\title{
Unruhiger Säugling mit Beinschmerzen und erhöhten Entzündungswerten
}

Das Majeed-Syndrom ist eine sehr seltene genetische Erkrankung, die sich mit einer kongenitalen dyserythropoetischen Anämie und Zeichen einer Osteomyelitis im Säuglingsalter manifestiert. Krankheitsverursachend ist eine Mutation im LPIN2Gen. Wir berichten hier über die erste uns bekannte Patientin in Deutschland mit dieser Erkrankung.

\section{Fallbericht}

\section{Anamnese}

Es erfolgte die Einweisung eines 10 Monate alten weiblichen Säuglings türkischstämmiger Eltern mit seit der Geburt auftretenden Unruhezuständen und erhöhten Entzündungswerten im Blut.

Das Mädchen habe schon immer viel getragen werden müssen, ein Ablegen des Säuglings war auch im schlafenden Zustand nur kurz möglich gewesen. Ab dem 4. Lebensmonat war es kurzzeitig zu einer leichten Besserung der Symptomatik gekommen. Nun vermuteten die Eltern erneut Schmerzen in den Beinen. Fieber war bisher nicht aufgetreten. In den vom niedergelassenen Kinderarzt veranlassten Laboruntersuchungen wurde ein erhöhtes $C$-reaktives Protein (CRP) von $8,7 \mathrm{mg} / \mathrm{dl}$ (Normwert<0,3 mg/ $\mathrm{dk}$ ) und ein erniedrigtes Hämoglobin $(\mathrm{Hb})$ von 9,3 g/dl (Normwert 10,7-13,1 g/dl) festgestellt.

\section{Untersuchungsbefund}

Im klinischen Untersuchungsbefund bei Aufnahme zeigte sich ein Säugling in gutem Allgemein- und zartem Ernährungszustand mit Mikrozephalie. Körpergewicht $7465 \mathrm{~g}$ (4. Perzentile), Körperlänge $71,7 \mathrm{~cm}$ (21. Perzentile), Kopfumfang 43,2 cm (1. Perzentile), Körpertemperatur $37,5^{\circ} \mathrm{C}$. Der pädiatrisch-internistische Zustand war ohne pathologischen Befund. Es zeigte sich eine diskrete Schonhaltung des linken Beines und eine Bewegungseinschränkung des linken Hüftgelenks.

\section{Labor}

Folgende Laborwerte waren bei Aufnahme pathologisch: $\mathrm{Hb} 9,2 \mathrm{~g} / \mathrm{dl}$ (Normwert 10,7-13,1 g/dl), MCV 68 fl (Normwert 73-101 fl), Thrombozyten 784/nl (Normwert 210-500/nl), CRP 8,2 mg/dl (Norm-
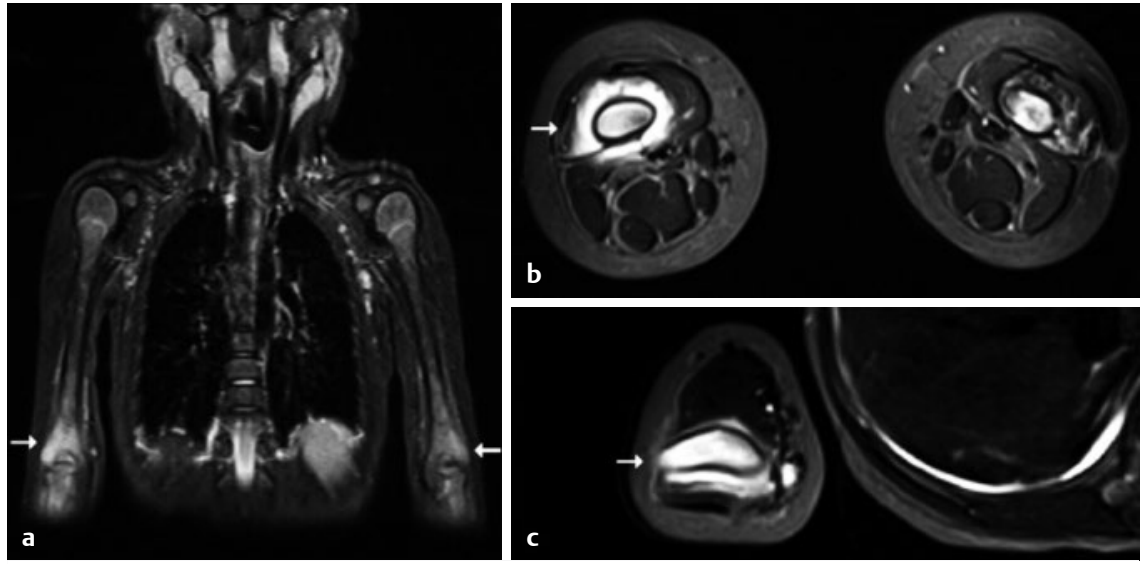

Abb. 1 Ganzkörper-MRT mit Zeichen einer nichtbakteriellen Osteomyelitis: vorwiegend medulläre und subperiostale, weniger weichteilbetreffende short-tau inversion recovery (STIR) Signalanhebungen (Pfeile) im a Humerus beidseits (coronar), b Femur diametaphysär rechts (transversal), c Olekranon rechts (transversal).

wert $<0,3 \mathrm{mg} / \mathrm{dl}$ ), Blutsenkungsgeschwindigkeit $120 \mathrm{~mm} / \mathrm{h}$ (Normwert<20 mm/h), Ferritin $121 \mathrm{ng} / \mathrm{ml}$ (Normwert 2-100 ng/ $\mathrm{ml}$ ). Normwertig waren: Differenzialblutbild, MCH, GOT, GPT, Kreatinin, Albumin, LDH, AP, Lipase, Prokalzitonin, Kreatininkinase, Vitamine $C$ und D, Parathormon, ANA (IFT), ds-DNA-Antikörper (Blot).

\section{Diagnostik}

Die Magnetresonanztomografie (MRT) der unteren Extremitäten ergab Befunde vereinbar mit einer Osteomyelitis des distalen Femur beidseits. Es erfolgten eine Knochenbiopsie mit mikrobiologischer und histopathologischer Untersuchung und der Beginn einer kalkulierten antibiotischen Therapie mit Cefuroxim intravenös. Die Histopathologie erbrachte ein in Kortikalis, Fettmark und Muskulatur lokalisiertes, gemischt zelluläres, eosinophil und lymphoplasmazelluläres Infiltrat, vereinbar mit dem Bild einer chronischen Osteomyelitis. Mittels Kultur und eubakterieller PCR gelang kein Keimnachweis. Im kurzfristigen Verlauf kam es unter der antibiotischen Therapie und Ibuprofen zu einer klinischen und laborchemischen Besserung.

Sieben Wochen nach der Biopsie traten erneut Unruhephasen mit einer CRP-Erhöhung auf. Daraufhin erfolgte eine Ganzkörper-MRT sowie - aufgrund der mikrozytären Anämie und zum Ausschluss einer hämatologischen Grunderkrankung - eine Knochenmarksaspiration.
In der Ganzkörper-MRT ( $\triangleright$ Abb. 1) zeigten sich medulläre und subperiostale, teilweise weichteilbetreffende Signalanhebungen im distalen Femur rechts, im Humerus beidseits, in der Ulna - vor allem im Olekranon - beidseits und im Radius beidseits. Der bildgebende Befund war somit vereinbar mit einer chronisch nichtbakteriellen Osteomyelitis (CNO). Allerdings war das Alter für diese Diagnose sehr untypisch.

Der Knochenmarksbefund zeigte eine deutlich gesteigerte dysplastische Erythropoese passend zu einer kongenitalen dyserythropoetischen Anämie ( $\triangleright$ Abb. 2).

\section{Diagnose und Verlauf}

In Zusammenschau der Befunde mit einer multifokalen Osteomyelitis und dyserythropoetischen Anämie wurde die Verdachtsdiagnose eines Majeed-Syndroms gestellt. In der Exomsequenzierung im Rahmen des TRANSLATE-NAMSE Projekts (Standort Ludwig-Maximilians-Universität München) wurde eine bisher nicht beschriebene homozygote splice site Deletionsvariante im LPIN2 Gen (c.2442 + 3_2442 + 6del) nachgewiesen. Die konsanguinen Eltern zeigten jeweils eine heterozygote Trägerschaft dieser LPIN2-Variante. Somit konnte gut vereinbar mit dem klinischen, laborchemischen, histologischen und bildgebenden Krankheitsbild die Diagnose eines Majeed-Syndroms genetisch gesichert werden. 


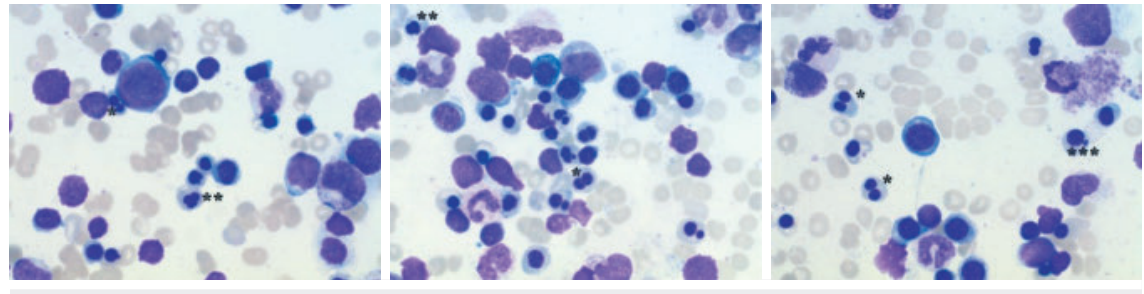

- Abb. 2 Knochenmarksausstrich mit gesteigerter Erythropoese, Doppel- $\left({ }^{*}\right)$ und Kleeblattkernformen $\left({ }^{* *}\right)$ und $\left({ }^{* * *}\right)$ Kernabsprengungen in den Normoblasten und vereinzelt Zytoplasmabrücken in den polychromatischen Erythroblasten.

Mit Ibuprofen $30 \mathrm{mg} / \mathrm{kg} / \mathrm{d}$ konnte zunächst klinisch ein gutes Ansprechen bei unserer Patientin erzielt werden. Im Verlauf präsentierte sich das Mädchen aber weiter mit anhaltenden Unruhezuständen und Erhöhung der Entzündungswerte, sodass eine Therapie mittels IL-1-Rezeptor-Blockade (Canakinumab) eingeleitet wurde.

\section{Diskussion}

Das Majeed-Syndrom ist eine autosomalrezessiv vererbte monogenetische autoinflammatorische Erkrankung und wurde erstmalig 1989 von Majeed et al. beschrieben [8]. Es gehört zu den seltenen Erkrankungen, und bisher wurden nur wenige pädiatrische Fallberichte publiziert (1, 3, 4, 6-12).

Betroffene Kinder entwickeln eine im Säuglings- oder Kleinkindalter auftretende chronische nichtbakterielle Osteomyelitis (CNO) und eine kongenitale dyserythropoetische Anämie (CDA). Zudem kann eine neutrophile Dermatose auftreten, wobei die Penetranz der Dermatose im Gegensatz zur stets vorhandenen CNO und CDA inkomplett ist [11].

Die Erkrankung wird durch autosomal rezessive pathogene Varianten im LPIN2Gen hervorgerufen [2]. LPIN2 kodiert für die Phosphatase Lipin-2, welche im Lipidmetabolismus von Makrophagen eine Rolle spielt. Dabei konnte Lipin-2 als wichtiger Akteur in der Regulierung des NLRP3Inflammasoms und der dadurch kontrollierten Produktion des proinflammatorischen Zytokins Interleukin-1 $\beta$ (IL-1 $\beta$ ) identifiziert werden [5].

Die Behandlung erfolgte meist empirisch mit NSAR und Kortikosteroiden. Hierunter konnte bei den meisten Patienten aller- dings keine komplette Symptomfreiheit erzielt werden. Weitere Therapieversuche mit Colchicin, Methotrexat, Etanercept oder Bisphosphonat - mit unterschiedlichen $\mathrm{Er}$ gebnissen - sind beschrieben. Bei unzureichender Therapie traten im Verlauf auch eine ausgeprägte Gedeihstörung und Gelenkdeformationen auf $[6,11,12]$.

In 4 neueren Fallberichten wurde eine Therapie mit einem IL-1-Rezeptor-Blocker eingeleitet, worunter ein sehr gutes Ansprechen zu verzeichnen war $[1,3,4,12]$.

Trotz monogenetischer Ätiologie des Majeed-Syndroms zeigten die bisher publizierten Fälle eine ausgeprägte Variabilität in der Schwere der Erkrankung. So berichten Roy et al. [12] über eine Familie bestehend aus konsanguinen Eltern und ihren 4 Kindern, die allesamt homozygote Träger einer pathogenen LPIN2-Variante waren. Allerdings waren nur 3 der Kinder klinisch schwer betroffen. Das 4. Kind und die Mutter wiesen leichte Symptome auf, der Vater war symptomfrei. In einem anderen Fall wird über einen 8 Monate alten Säugling berichtet, der unter rezidivierendem Fieber, leichter Anämie und ausgeprägter Neutropenie litt. Eine Osteomyelitis lag klinisch nicht vor. Molekulargentisch wurde eine compound heterozygote Mutation im LPIN2-Gen nachgewiesen [4].

Diese variable Penetranz und Expressivität wurde auch von Rao et al. [11] beschrieben. In ihrem Fallbericht aus Indien von 2 Cousins mit der gleichen pathogenen LPIN2Variante hatte nur einer der beiden eine früh auftretende CNO mit CDA. Der 2. Patient hingegen wies einen milden Verlauf auf, passend zu einer nicht syndromalen CNO.

Diese Variabilität in der klinischen Erscheinung des Majeed-Syndroms mit auch mil- den Verlaufsformen ohne Fieber oder CNO lassen eine höhere Prävalenz eines gegebenenfalls atypischen Majeed-Syndroms als bisher angenommen vermuten. Somit sollte klinisch das Majeed-Syndrom nicht nur als Differenzialdiagnose einer multifokalen Osteomyelitis im Säuglings- und Kleinkindalter mit Anämie, sondern auch bei therapierefraktären Verläufen einer juvenilen CRMO und bei Fieber unklarer Genese Beachtung finden.

\section{FAZIT FÜR DIE PRAXIS}

- Bei anhaltenden Unruhezuständen und erhöhten nicht infektionsbedingten Entzündungswerten sollte im Säuglingsalter auch an eine aseptische Osteomyelitis gedacht werden.

- Bei zusätzlich vorliegender dyserythropoetischer Anämie ist die Diagnose eines Majeed-Syndroms zu erwägen. Eine „klassische" CRMO tritt nicht vor dem 6. Lebensjahr auf.

- Diagnoserelevant ist die genetische Untersuchung des LPIN2Gens.

- Bei schweren Verläufen ist eine Therapie mit IL-1-RezeptorBlockern vielversprechend.

- Bei Verdacht auf eine autoinflammatorische Störung kann grundsätzlich eine frühzeitige (Trio-)Exomsequenzierung bei der Diagnosestellung helfen.

\section{Interessenkonflikt}

Die Autorinnen und Autoren geben an, dass kein Interessenkonflikt besteht.

\section{Einhaltung ethischer Richtlinien}

Die Autorinnen und Autoren bestätigen, dass eine schriftliche Einwilligung der Eltern der Patientin zur Publikation dieses Fallberichts vorliegt. 
Fabian Glowatzki' ${ }^{1}$, Julia Eilenberger ${ }^{2,3}$ Friederike Blankenburg ${ }^{1}$, Christian Stirnkorb ${ }^{1}$, Aleš Janda ${ }^{4}$, Thekla von Kalle $^{5}$, Ute Gross-Wieltsch ${ }^{6}$, Fabian Hauck $^{2,3,7}$, Toni Hospach ${ }^{1}$

'Zentrum für Pädiatrische Rheumatologie im Olgahospital Stuttgart, Klinikum Stuttgart

${ }^{2}$ Kinderklinik und Kinderpoliklinik im Dr. von Haunerschen Kinderspital, Klinikum der Universität München, Ludwig-Maximilians-Universität München

${ }^{3}$ Münchner Zentrum für Seltene Erkrankungen, Care for Rare Center am Dr. von Haunerschen Kinderspital, Klinikum der Universität München, Ludwig-MaximiliansUniversität München

4Klinik für Kinder- und Jugendmedizin, Universitätsklinikum Ulm

${ }^{5}$ Kinderradiologisches Institut im Olgahospital Stuttgart, Klinikum Stuttgart

${ }^{6}$ Pädiatrische Onkologie, Hämatologie und Immunologie im Olgahospital Stuttgart, Klinikum Stuttgart

${ }^{7}$ Deutsches Zentrum für Infektionsforschung, Standort Ludwig-MaximiliansUniversität München

\section{Korrespondenzadresse}

Dr. med. Toni Hospach

Zentrum für Pädiatrische Rheumatologie am Klinikum Stuttgart (ZEPRAS)

Klinikum Stuttgart gKAöR

Olgahospital, Pädiatrie 2

Kriegsbergstr. 62, 70174 Stuttgart

A.Hospach@klinikum-stuttgart.de

\section{Literatur}

[1] Al MZ, Madan W, Al MB et al. Dramatic Response of Familial Majeed Syndrome to Interleukin-1 Antagonist Therapy: Case report. Arch Rheumatol 2019; 34: 352-356. doi:10.5606/ArchRheumatol.2019.7267

[2] Ferguson PJ, Chen S, Tayeh MK et al. Homozygous mutations in LPIN2 are responsible for the syndrome of chronic recurrent multifocal osteomyelitis and congenital dyserythropoietic anaemia (Majeed syndrome). J Med Genet 2005; 42: 551-557. doi:10.1136/jmg.2005.030759

[3] Herlin TB, Fiirgaard MB, Kerndrup G et al. Efficacy of anti-IL-1 treatment in Majeed syndrome. Ann Rheum Dis 2013; 72: 410-413. doi:10.1136/annrheumdis-2012-201818

[4] Liu J, X. Hu XY, Zhao ZP et al. Compound heterozygous LPIN2 pathogenic variants in a patient with Majeed syndrome with recurrent fever and severe neutropenia: case report. BMC Med Genet 2019; 20: 182. doi:10.1186/s12881-019-0919-3

[5] Lorden G, Sanjuan-Garcia I, de Pablo N et al. Lipin-2 regulates NLRP3 inflammasome by affecting P2X7 receptor activation. J Exp Med 2017; 214: 511-528. doi:10.1084/ jem.20161452

[6] Majeed HA, Al-Tarawna M, El-Shanti M et al. The syndrome of chronic recurrent multifocal osteomyelitis and congenital dyserythropoietic anaemia. Report of a new family and a review. Eur J Pediatr 2001; 160 : 705-710

[7] Majeed HA, El-Shanti H, Al-Rimawi H, Al-Masri N. On mice and men: An autosomal recessive syndrome of chronic recurrent multifocal osteomyelitis and congenital dyserythropoietic anemia. J Pediatr 2000; 137: 441-442. doi:10.1067| mpd.2000.107613
[8] Majeed HA, Kalaawi M, Mohanty D et al. Congenital dyserythropoietic anemia and chronic recurrent multifocal osteomyelitis in three related children and the association with Sweet syndrome in two siblings. I Pediatr 1989; 115: 730-734. doi:10.1016| s0022-3476(89)80650-x

[9] Moussa T, Bhat V, Kini V, Fathalla BM. Clinical and genetic association, radiological findings and response to biological therapy in seven children from Qatar with non-bacterial osteomyelitis. Int J Rheum Dis 2017; 20: 1286-1296. doi:10.1111/1756185X.12940

[10] Pinto-Fernandez C., Seoane-Reula ME. Efficacy of treatment with IL-1RA in Majeed syndrome. Allergol Immunopathol (Madr) 2017; 45: 99-101. doi:10.1016/j. aller.2016.04.003

[11] Rao AP, Gopalakrishna DB, Bing X, Ferguson PJ. Phenotypic Variability in Majeed Syndrome. J Rheumatol 2016; 43: 1258-1259. doi:10.3899/jrheum.151193

[12] Roy NB, Zaal Al, Hall G et al. Majeed syndrome: description of a novel mutation and therapeutic response to bisphosphonates and IL-1 blockade with anakinra. Rheumatology (Oxford) 2019. doi:10.1093/ rheumatology/kez317

Bibliografie

DOI https://doi.org/10.1055/a-1098-6907 arthritis + rheuma 2020; 40: 124-126 (c) Georg Thieme Verlag KG Stuttgart · New York ISSN 0176-5167 